\title{
Frame expansions of test functions, tempered distributions, and ultradistributions
}

\author{
Stevan Pilipović ${ }^{a}$ and Diana T. Stoeva ${ }^{b}$ \\ ${ }^{a}$ Department of Mathematics and Informatics, University of Novi Sad, \\ Trg D. Obradovića 4, 21000 Novi Sad, Serbia, pilipovic@im.ns.ac.yu \\ ${ }^{b}$ Acoustics Research Institute, Austrian Academy of Sciences, \\ Wohllebengasse 12-14, Vienna 1040, Austria, dstoeva@kfs.oeaw.ac.at
}

November 12, 2018

\begin{abstract}
The paper is devoted to frame expansions in Fréchet spaces. First we review some results which concern series expansions in general Fréchet spaces via Fréchet and General Fréchet frames. Then we present some new results on series expansions of tempered distributions and ultradistributions, and the corresponding test functions, via localized frames and coefficients in appropriate sequence spaces.
\end{abstract}

Keywords: Tempered distributions and ultradistributions, Frame expansions, Fréchet frames, Localized frames

$M S C$ 2010: 42C15, 46F05

\section{Introduction}

In this paper we present results devoted to frame expansions in Fréchet spaces. First we consider the general case, expansions via Fréchet frames and appropriate dual sequences in general, and then we aim expansions of generalized functions via a proper class of frames. As the Hermite expansions are the basic ones for tempered distributions and ultradistributions, a suitable localization of a frame with respect to the Hermite basis enables us to extend the consideration of generalized functions using appropriate class of frames instead of the Hermite basis. Frames were introduced in Hilbert spaces [8]. They generalize the concept of an orthonormal basis allowing even redundancy, but still provide series expansions of all the elements of the space. Frames were extended to Banach spaces (atomic decompositions and Banach frames [14, 15, 16], p-frames [1, $X_{d}$-frames [5]) and furthermore to Fréchet spaces (pre-Fréchet, Fréchet, and General Frechet frames, [20, 21, 22]). 
While Hilbert frames always guarantee series expansions in Hilbert spaces, this is not always the case with Banach and Fréchet frames. In this paper we review some results related to sufficient conditions for series expansions in general Fréchet spaces, as well as present new results devoted to series expansions in certain spaces of test functions and their duals via appropriate frames. The paper is organized as follows. Section 2 contains the main definitions, notation, and basic needed facts. In Section 3 we review some results from [21, 22] which are related to series expansions in general Fréchet spaces via Fréchet and General Fréchet frames. Section 4 is devoted to new results which concern expansions in certain spaces of test functions, tempered distributions, and ultradistributions, via localized frames; the statements are given without proofs and the proofs are subject of a further extended paper [23].

\section{Preliminaries}

Throughout the paper, $(H,\langle\cdot, \cdot\rangle)$ denotes a separable Hilbert space. We consider countable sequences and for convenience of the writing we index them by the set $\mathbb{N}$. A sequence $\left(g_{n}\right)_{n=1}^{\infty}$ with elements from $H$ is called: a frame for $H$ if there exist positive constants $A$ and $B$ (called frame bounds) so that $A\|f\|^{2} \leq \sum_{n=1}^{\infty}\left|\left\langle f, g_{n}\right\rangle\right|^{2} \leq$ $B\|f\|^{2}$ for every $f \in H$ [8]; a Riesz basis for $H$ if its elements are the images of the elements of an orthonormal basis under a bounded bijective operator on $H$ [4. Let us recall some needed basic facts from frame theory (see e.g [7]). Let $G=\left(g_{n}\right)_{n=1}^{\infty}$ be a frame for $H$. Then there exists a frame $\left(f_{n}\right)_{n=1}^{\infty}$ for $H$ so that $f=\sum_{n=1}^{\infty}\left\langle f, f_{n}\right\rangle g_{n}=\sum_{n=1}^{\infty}\left\langle f, g_{n}\right\rangle f_{n}, f \in H$. Such $\left(f_{n}\right)_{n=1}^{\infty}$ is called a dual frame of $\left(g_{n}\right)_{n=1}^{\infty}$. The analysis operator $U_{G}$, given by $U_{G} f=\left(\left\langle f, g_{n}\right\rangle\right)_{n=1}^{\infty}$, is bounded from $H$ into $\ell^{2}$, the synthesis operator $T_{G}$ defined on the finite sequences by $T_{G}(c)=\sum_{n} c_{n} e_{n}$ extends to a bounded operator from $\ell^{2}$ into $H$, the frame operator $S_{G}=T_{G} U_{G}$ is a bounded bijection of $H$ onto $H$, and the series in $S_{G} f=\sum_{n=1}^{\infty}\left\langle f, g_{n}\right\rangle g_{n}$ converges unconditionally. The sequence $\left(S_{G}^{-1} g_{n}\right)_{n=1}^{\infty}$ is a dual frame of $\left(g_{n}\right)_{n=1}^{\infty}$, called the canonical dual of $\left(g_{n}\right)_{n=1}^{\infty}$, and it will be denoted by $\left(\widetilde{g_{n}}\right)_{n=1}^{\infty}$. When $\left(g_{n}\right)_{n=1}^{\infty}$ is a Riesz basis of $H$ (and thus a frame for $H$ ), then $\left(\widetilde{g_{n}}\right)_{n=1}^{\infty}$ is the only dual frame of $\left(g_{n}\right)_{n=1}^{\infty}$. Frames which are not Riesz bases have other dual frames in addition to the canonical dual. Recall also the localization-notions introduced in [17]. Given a Riesz basis $\left(g_{n}\right)_{n=1}^{\infty}$ for $H$, a frame $E=\left(e_{n}\right)_{n=1}^{\infty}$ for $H$ is called: polynomially localized with respect to $\left(g_{n}\right)_{n=1}^{\infty}$ with decay $s>0$ if there is a constant $C_{s}>0$ so that $\max \left\{\left|\left\langle e_{m}, g_{n}\right\rangle\right|,\left|\left\langle e_{m}, \widetilde{g_{n}}\right\rangle\right|\right\} \leq C_{s}(1+|m-n|)^{-s}, m, n \in \mathbb{N}$; exponentially localized with respect to $\left(g_{n}\right)_{n=1}^{\infty}$ if for some $s>0$ there is a constant $C_{s}>0$ so that $\max \left\{\left|\left\langle e_{m}, g_{n}\right\rangle\right|,\left|\left\langle e_{m}, \tilde{g_{n}}\right\rangle\right|\right\} \leq C_{s} \mathrm{e}^{-s|m-n|}, m, n \in \mathbb{N}$. Notice that in the literature there exist other ways to define localization of frames [2, 3, 13, 12, but for the purpoces of the current paper it is relevant and enough to use the localization concepts according to [17].

Next, $(X,\|\cdot\|)$ denotes a Banach space and $(\Theta,\||\cdot|\|)$ denotes a Banach sequence space. A Banach sequence space is a $B K$-space if the coordinate functionals are 
continuous. If the canonical vectors form a Schauder basis for $\Theta$, then $\Theta$ is called a $C B$-space and it is clearly a $B K$-space. Given a $B K$-space $\Theta$ and a Riesz basis $G=\left(g_{n}\right)_{n=1}^{\infty}$ for $H$, one associates to $\Theta$ the following Banach space

$\mathfrak{H}_{G}^{\Theta}:=\left\{f \in H: f=\sum_{n=1}^{\infty} c_{n} g_{n}\right.$ with $\left.\left(c_{n}\right)_{n=1}^{\infty} \in \Theta\right\}$ normed by $\|f\|_{\mathfrak{H}_{G}^{\Theta}}:=\left\|\left(c_{n}\right)_{n=1}^{\infty}\right\|_{\Theta}$.

Further, we consider Fréchet spaces which are projective limits of Banach spaces. Let $\left\{Y_{k},|\cdot|_{k}\right\}_{k \in \mathbb{N}_{0}}$ be a sequence of separable Banach spaces such that

$$
\begin{gathered}
\{\mathbf{0}\} \neq \cap_{k \in \mathbb{N}_{0}} Y_{k} \subseteq \ldots \subseteq Y_{2} \subseteq Y_{1} \subseteq Y_{0} \\
|\cdot|_{0} \leq|\cdot|_{1} \leq|\cdot|_{2} \leq \ldots \\
Y_{F}:=\cap_{k \in \mathbb{N}_{0}} Y_{k} \text { is dense in } Y_{k}, k \in \mathbb{N}_{0} .
\end{gathered}
$$

Under the conditions (1)-(3), $Y_{F}$ is a Fréchet space with the sequence of norms $|\cdot|_{s}$, $s \in \mathbb{N}_{0}$, and it is called the projective limit of $Y_{k}, k \in \mathbb{N}_{0}$. We will use such type of sequences in two cases:

1. $Y_{k}=X_{k}$ with norm $\|\cdot\|_{k}, k \in \mathbb{N}_{0}$;

2. $Y_{k}=\Theta_{k}$ with norm $\|\cdot \cdot\|_{k}, k \in \mathbb{N}_{0}$.

We use the therm operator for a linear mapping. Given sequences of Banach spaces, $\left\{X_{k}\right\}_{k \in \mathbb{N}_{0}}$ and $\left\{\Theta_{k}\right\}_{k \in \mathbb{N}_{0}}$, which satisfy (11)-(3), an operator $G: \Theta_{F} \rightarrow X_{F}$ is called F-bounded if for every $k \in \mathbb{N}_{0}$, there exists a constant $C_{k}>0$ such that $\left\|G\left(c_{n}\right)_{n=1}^{\infty}\right\|_{k} \leq C_{k}\|\|\left\{c_{n}\right\}_{n=1}^{\infty} \|_{k}$ for all $\left(c_{n}\right)_{n=1}^{\infty} \in \Theta_{F}$. Now we recall the definitions of Fréchet and General Fréchet frames.

Definition 2.1 [22] Let $\left\{X_{k},\|\cdot\|_{k}\right\}_{k \in \mathbb{N}_{0}}$ be a sequence of Banach spaces satisfying (11)-(3) and let $\left\{\Theta_{k},\|\cdot \mid\|_{k}\right\}_{k \in \mathbb{N}_{0}}$ be a sequence of BK-spaces satisfying (1)-(3). A sequence $\left(g_{n}\right)_{n=1}^{\infty}$ with elements from $X_{F}^{*}$ is called: a General pre-Fréchet frame (in short, General pre-F-frame) for $X_{F}$ with respect to $\Theta_{F}$ if there exist sequences $\left\{\widetilde{s}_{k}\right\}_{k \in \mathbb{N}_{0}} \subseteq \mathbb{N}_{0},\left\{s_{k}\right\}_{k \in \mathbb{N}_{0}} \subseteq \mathbb{N}_{0}$, which increase to $\infty$ with the property $s_{k} \leq \widetilde{s}_{k}$, $k \in \mathbb{N}_{0}$, and there exist constants $0<A_{k} \leq B_{k}<\infty, k \in \mathbb{N}_{0}$, satisfying

$$
\begin{gathered}
\left(g_{n}(f)\right)_{n=1}^{\infty} \in \Theta_{F}, f \in X_{F}, \\
A_{k}\|f\|_{s_{k}} \leq\|\|\left\{g_{n}(f)\right\}_{n=1}^{\infty} \mid\left\|_{k} \leq B_{k}\right\| f \|_{\widetilde{s}_{k}}, f \in X_{F}, k \in \mathbb{N}_{0}
\end{gathered}
$$

a General Fréchet frame (in short, General $F$-frame) for $X_{F}$ with respect to $\Theta_{F}$ if it is a General pre-F-frame for $X_{F}$ with respect to $\Theta_{F}$ and there exists a continuous operator $V: \Theta_{F} \rightarrow X_{F}$ so that $V\left(g_{n}(f)\right)_{n=1}^{\infty}=f$ for every $f \in X_{F}$.

Let $s_{k}=\widetilde{s}_{k}=k, k \in \mathbb{N}_{0}$. In this case the above definition of a General pre$F$-frame reduces to the definition of a pre-Fréchet frame (in short pre-F-frame) [20], and if in addition the continuity of $V$ is replaced by the stronger condition of $F$-boundedness of $V$, then one comes to the concept of a Fréchet frame (in short 
F-frame) [21. In the particular case when $X_{k}=X$, and $\Theta_{k}=\Theta, k \in \mathbb{N}_{0}$ : an $F$ frame (resp. pre- $F$-frame) for $X_{F}$ with respect to $\Theta_{F}$ is actulally a Banach frame for $X$ with respect to $\Theta$ (resp. $\Theta$-frame for $X$ ) as introduced in [16] (resp. [5]); when (4) and the upper inequality of (5) hold, one comes to the definition of a $\Theta$-Bessel sequence for $X$.

When $\left(g_{n}\right)_{n=1}^{\infty}$ is a pre-F-frame for $X_{F}$ with respect to $\Theta_{F}$, then for any $n \in \mathbb{N}$ and any $k \in \mathbb{N}_{0}, g_{n}$ can be extended in a unique way to a continues operator on $X_{k}$ and this extension will be denoted by $g_{n}^{k}$.

Recall that a positive continuous function $\mu$ on $\mathbb{R}$ is called: a $k$-moderate weight if $k \geq 0$ and there exists a constant $C>0$ so that $\mu(t+x) \leq C(1+|t|)^{k} \mu(x), t, x \in \mathbb{R}$; a sub-exponential weight, if there exist constants $C>0, \gamma>0$ and $\beta \in(0,1)$ so that $\mu(t+x) \leq C e^{\gamma|t|^{\beta}} \mu(x), t, x \in \mathbb{R}$. Taking $\mu_{k}(x)=(1+|x|)^{k}$ (resp. $\beta \in(0,1)$ and $\left.\mu_{k}(x)=e^{k|x|^{\beta}}\right), x \in \mathbb{R}, k \in \mathbb{N}_{0}$, the spaces $\Theta_{k}:=\ell_{\mu_{k}}^{2}, k \in \mathbb{N}_{0}$, satisfy (10)-(3) and their projective limit $\cap_{k} \Theta_{k}$ is the so called space of rapidly decreasing sequences $\mathbf{s}$ (resp. space of sub-exponentially decreasing sequences $\mathfrak{s}^{\beta}$ ). Further, we will use the following statement:

Lemma 2.2 Let $G=\left(g_{n}\right)_{n=1}^{\infty}$ be a Riesz basis for $H$. For $k \in \mathbb{N}_{0}$, let $\mu_{k}$ be a $k$-moderate weight so that $1=\mu_{0}(x) \leq \mu_{1}(x) \leq \mu_{2}(x) \leq \ldots$, for every $x \in \mathbb{R}$. Then $\left\{\Theta_{k}\right\}_{k \in \mathbb{N}_{0}}:=\left\{\ell_{\mu_{k}}^{2}\right\}_{k \in \mathbb{N}_{0}}$ is a sequence of $C B$-spaces satisfying (11)-(3), the spaces $X_{k}:=\mathfrak{H}_{G}^{\Theta_{k}}, k \in \mathbb{N}_{0}$, satisfy (11)-(3), and $g_{n} \in X_{F}$ for every $n \in \mathbb{N}$. The conclusions also hold if the assumptions " $\mu_{k}-k$-moderate weight" are replaced by " $\mu_{k}-$ sub-exponential weight".

Test function spaces and their duals under consideration in the paper are: $\mathcal{S}(\mathbb{R}):=\left\{f \in C^{\infty}(\mathbb{R}):|f|_{k}:=\sup _{x \in \mathbb{R}} \sup _{m \leq k}\left|f^{(m)}(x)\right|\left(1+|x|^{2}\right)^{k / 2}<\infty, \quad \forall k \in\right.$ $\left.\mathbb{N}_{0}\right\}$, and its dual $\mathcal{S}^{\prime}(\mathbb{R}) \subset C^{\infty}(\mathbb{R})$, the space of tempered distributions;

$\Sigma^{\alpha}:=\left\{\phi \in C^{\infty}(\mathbb{R}):|\phi|_{h, \alpha}:=\sup _{n \in \mathbb{N}_{0}, x \in \mathbb{R}} \frac{h^{n} e^{m|x|^{1 / \alpha}\left|\phi^{(n)}(x)\right|}}{n !^{\prime \alpha}}<\infty, \forall h>0\right\}$, and its dual $\left(\Sigma^{\alpha}(\mathbb{R})\right)^{\prime}, \alpha>1 / 2$, the space of Beurling tempered ultradistributions, cf. [19, 11, 6].

In the sequel, $\left(h_{n}\right)_{n=1}^{\infty}$ is the Hermite orthonormal basis $\left(\mathbf{h}_{n}\right)_{n=0}^{\infty}$ of $L^{2}(\mathbb{R})$, reindexed from 1 to $\infty$, i.e., $h_{n+1}(t)=\mathbf{h}_{n}(t)=\left(2^{(n)} n ! \sqrt{\pi}\right)^{-1 / 2}(-1)^{n} e^{t^{2} / 2} \frac{d^{n}}{d t^{n}}\left(e^{-t^{2}}\right)$, $n \in \mathbb{N}_{0}$. Recall that $h_{n} \in \mathcal{S}$ and $h_{n} \in \Sigma^{\alpha}, \alpha>1 / 2, n \in \mathbb{N}$. Moreover, we know [24] the following:

- If $f \in \mathcal{S}$, then $\left(\left\langle f, h_{n}\right\rangle\right)_{n=1}^{\infty} \in \mathbf{s}$; conversely, if $\left(a_{n}\right)_{n=1}^{\infty} \in \mathbf{s}$, then $\sum_{n=1}^{\infty} a_{n} h_{n}$ converges in $\mathcal{S}$ to $f=\sum_{n=1}^{\infty}\left\langle f, h_{n}\right\rangle h_{n},\left(\left\langle f, h_{n}\right\rangle\right)_{n=1}^{\infty}=\left(a_{n}\right)_{n=1}^{\infty}$.

- If $F \in \mathcal{S}^{\prime}$, then $\left(b_{n}\right)_{n=1}^{\infty}:=\left(F\left(h_{n}\right)\right)_{n=1}^{\infty} \in \mathbf{s}^{\prime}$ and $F(f)=\sum_{n=1}^{\infty}\left\langle f, h_{n}\right\rangle b_{n}, f \in \mathcal{S}$; conversely, if $\left(b_{n}\right)_{n=1}^{\infty} \in \mathbf{s}^{\prime}$, then the mapping $F: f \rightarrow \sum_{n=1}^{\infty}\left\langle f, h_{n}\right\rangle b_{n}$ is well defined on $\mathcal{S}$, it determines $F$ as an element of $\mathcal{S}^{\prime}$ and $\left(F\left(h_{n}\right)\right)_{n=1}^{\infty}=\left(b_{n}\right)_{n=1}^{\infty}$.

The above two statements also hold when $\mathcal{S}, \mathcal{S}^{\prime}, \mathbf{s}$, and $\mathbf{s}^{\prime}$ are replaced by $\Sigma^{\alpha}$, $\left(\Sigma^{\alpha}\right)^{\prime}, \mathfrak{s}^{1 /(2 \alpha)}$, and $\left(\mathfrak{s}^{1 /(2 \alpha)}\right)^{\prime}$ with $\alpha>1 / 2$, respectively [19, 11, 6]. 


\section{Frame expansions in Fréchet spaces}

In this section we recall some general statements about sufficient conditions for series expansions in Fréchet spaces via Fréchet and General Fréchet frames, and appropriate dual sequences. We start with the case of Fréchet frames.

Proposition 3.1 [21] Let $\left(g_{n}\right)_{n=1}^{\infty}$ be an F-frame for $X_{F}$ with respect to $\Theta_{F}$. Then the following holds.

(a) For every $k \in \mathbb{N}_{0}$, the sequence $\left\{g_{i}^{k}\right\}_{i=1}^{\infty}$ is a Banach frame for $X_{s}$ with respect to $\Theta_{k}$.

(b) If $\Theta_{k}, k \in \mathbb{N}_{0}$, are CB-spaces, then there exists $\left(f_{n}\right)_{n=1}^{\infty} \in\left(X_{F}\right)^{\mathbb{N}}$, which is $\Theta_{k}^{*}$-Bessel sequence for $X_{k}^{*}$ for every $k \in \mathbb{N}_{0}$ and such that

$$
\begin{aligned}
f & =\sum_{i=1}^{\infty} g_{i}(f) f_{i}, f \in X_{F}, \quad\left(\text { in } X_{F}\right), \\
g & =\sum_{i=1}^{\infty} g\left(f_{i}\right) g_{i}, g \in X_{F}^{*}, \quad\left(\text { in } X_{F}^{*}\right), \\
f & =\sum_{i=1}^{\infty} g_{i}^{k}(f) f_{i}, f \in X_{k}, k \in \mathbb{N}_{0} .
\end{aligned}
$$

(c) If $\Theta_{k}$ and $\Theta_{k}^{*}, k \in \mathbb{N}_{0}$, are CB-spaces, then there exists $\left(f_{n}\right)_{n=1}^{\infty} \in\left(X_{F}\right)^{\mathbb{N}}$, which is a $\Theta_{k}^{*}$-frame for $X_{k}^{*}$ for every $k \in \mathbb{N}_{0}$ and such that (6)-(8) hold, and moreover,

$$
g=\sum_{i=1}^{\infty} g\left(f_{i}\right) g_{i}^{k}, g \in X_{k}^{*}, k \in \mathbb{N}_{0} .
$$

(d) If $\Theta_{k}, k \in \mathbb{N}_{0}$, are reflexive $C B$-spaces, then there exists $\left(f_{n}\right)_{n=1}^{\infty} \in\left(X_{F}\right)^{\mathbb{N}}$, which is a Banach frame for $X_{k}^{*}$ with respect to $\Theta_{k}^{*}$ for every $k \in \mathbb{N}_{0}$ such that (6)-(9) hold.

As one can see in Proposition 3.1, the F-boundedness property of $V$ leads to series expansions in all the spaces $X_{k}, k \in \mathbb{N}_{0}$. Now we continue with the case of General Fréchet frames and show that in this case the continuity property of $V$ is enough to imply the existence of a subsequence $\left\{X_{\widetilde{w}_{j}}\right\}_{j=0}^{\infty}$ of the given sequence $\left\{X_{\widetilde{s}_{k}}\right\}_{k=0}^{\infty}$ according to Definition 2.1, so that one has series expansions in $X_{\widetilde{w}_{j}}$, $j \in \mathbb{N}_{0}$, with convergence in appropriate norms.

Theorem 3.2 [22] Let $\left(g_{n}\right)_{n=1}^{\infty}$ be a General $F$-frame for $X_{F}$ with respect to $\Theta_{F}$ and let $\Theta_{k}, k \in \mathbb{N}_{0}$, be CB-spaces. Then there exist sequences $\left\{w_{j}\right\}_{j \in \mathbb{N}_{0}},\left\{r_{j}\right\}_{j \in \mathbb{N}_{0}}$, 
and $\left\{\widetilde{w}_{j}\right\}_{j \in \mathbb{N}_{0}}$, which increase to $\infty$ and there exist constants $\widetilde{A}_{j}, \widetilde{B}_{j}, j \in \mathbb{N}_{0}$, such that for every $j \in \mathbb{N}_{0}$,

$$
\widetilde{A}_{j}\|f\|_{w_{j}} \leq\|\|\left\{g_{i}(f)\right\}_{i=1}^{\infty}\|\|_{r_{j}} \leq \widetilde{B}_{j}\|f\|_{\widetilde{w}_{j}}, \forall f \in X_{F} .
$$

Moreover, there exists a sequence $\left(f_{n}\right)_{n=1}^{\infty} \in\left(X_{F}\right)^{\mathbb{N}}$ such that for every $j \in \mathbb{N}_{0}$, $\left(f_{n}\right)_{n=1}^{\infty}$ is a $\Theta_{r_{j}}^{*}$-Bessel sequence for $X_{w_{j}}^{*}$ and

$$
f=\sum_{i=1}^{\infty} g_{i}^{\widetilde{w}_{j}}(f) f_{i} \text { in }\|\cdot\|_{w_{j}} \text {-norm, } f \in X_{\widetilde{w}_{j}} .
$$

\section{Expansions of tempered distributions and ultradistributions by localized frames}

In this section, we aim expansions in a Fréchet space and its dual by localized frames and coefficients in a corresponding Fréchet sequence space. First we present a general result based on frames localized with respect to a Riesz basis and providing frame expansions in a corresponding Fréchet space, and then we apply it to obtain frame expansions in spaces of tempered distributions and ultradistributions, as well as in the corresponding test function spaces. To clarify some notation, when we take a frame element $e_{n} \in X_{F}\left(\subset H \subset X_{F}^{*}\right)$ and consider it as a functional in $X_{F}^{*}$, then we denote it by bold-style $\mathbf{e}_{\mathbf{n}}$.

Let us start with the general theorem about expansions in Fréchet spaces via localized frames.

Theorem 4.1 Let the assumptions and notation of Lemma 2.2 hold. Assume that $E=\left(e_{n}\right)_{n=1}^{\infty}$ is a sequence with elements from $X_{F}$ which is a frame for $H$ and polynomially localized with respect to $G$ with decay s for every $s \in \mathbb{N}$ (resp. exponentially localized with respect to $G$ ). Then the following statements hold.

(a) $\tilde{e_{n}} \in X_{F}$ for every $n \in \mathbb{N}$.

(b) The analysis operator $U_{E}$ is $F$-bounded from $X_{F}$ into $\Theta_{F}$, the synthesis operator $T_{E}$ is F-bounded from $\Theta_{F}$ into $X_{F}$, the frame operator $S_{E}$ is F-bounded and bijective from $X_{F}$ onto $X_{F}$ with unconditional convergence of the series in $S_{E} f=\sum_{n=1}^{\infty}\left\langle f, e_{n}\right\rangle e_{n}$, and $\widetilde{e_{n}} \in X_{F}, n \in \mathbb{N}$.

(c) For every $f \in X_{F}$,

$$
\begin{aligned}
& f=\sum_{n=1}^{\infty}\left\langle f, \widetilde{e_{n}}\right\rangle e_{n}=\sum_{n=1}^{\infty}\left\langle f, e_{n}\right\rangle \widetilde{e_{n}} \text { (with convergence in } X_{F} \text { ) } \\
& \text { with }\left(\left\langle f, \widetilde{e_{n}}\right\rangle\right)_{n=1}^{\infty} \in \Theta_{F} \text { and }\left(\left\langle f, e_{n}\right\rangle\right)_{n=1}^{\infty} \in \Theta_{F} .
\end{aligned}
$$

(d) If $X_{F}$ and $\Theta_{F}$ have the following property with respect to $\left(g_{n}\right)_{n=1}^{\infty}$ :

$$
\mathcal{P}_{\left(g_{n}\right)} \text { : For } f \in H \text {, one has } f \in X_{F} \text { if and only if }\left(\left\langle f, g_{n}\right\rangle\right)_{n=1}^{\infty} \in \Theta_{F} \text {. }
$$

then they also have the properties $\mathcal{P}_{\left(e_{n}\right)}$ and $\mathcal{P}_{\left(\widetilde{e_{n}}\right)}$. 
(e) Both $\left(\mathbf{e}_{\mathbf{n}}\right)_{n=1}^{\infty}$ and $\left(\widetilde{\mathbf{e}_{\mathbf{n}}}\right)_{n=1}^{\infty}$ form Fréchet frames for $X_{F}$ with respect to $\Theta_{F}$.

(f) For every $g \in X_{F}^{*}$,

$$
g=\sum_{n=1}^{\infty} g\left(e_{n}\right) \widetilde{\mathbf{e}_{\mathbf{n}}}=\sum_{n=1}^{\infty} g\left(\widetilde{e_{n}}\right) \mathbf{e}_{\mathbf{n}} \text { (with convergence in } X_{F}^{*} \text { ) }
$$

with $\left(g\left(e_{n}\right)\right)_{n=1}^{\infty} \in \Theta_{F}^{*}$ and $\left(g\left(\widetilde{e_{n}}\right)\right)_{n=1}^{\infty} \in \Theta_{F}^{*}$.

(g) If $\left(a_{n}\right)_{n=1}^{\infty} \in \Theta_{F}^{*}$, then $\sum_{n=1}^{\infty} a_{n} \mathbf{e}_{n}$ (resp. $\sum_{n=1}^{\infty} a_{n} \widetilde{\mathbf{e}_{\mathbf{n}}}$ ) converges in $X_{F}^{*}$, i.e., the mapping $f \mapsto \sum_{n=1}^{\infty}\left\langle f, e_{n}\right\rangle a_{n}$ (resp. $f \mapsto \sum_{n=1}^{\infty}\left\langle f, \tilde{e}_{n}\right\rangle a_{n}$ ) determines a continuous linear functional on $X_{F}$.

Remark: Note that in the setting of the above theorem, when $G$ is an orthonormal basis of $H$ or more generally, when $G$ is a Riesz basis for $H$ satisfying any of the following two conditions:

$\left(\mathcal{P}_{1}\right): \forall s \in \mathbb{N} \exists C_{s}>0:\left|\left\langle g_{m}, g_{n}\right\rangle\right| \leq C_{s}(1+|m-n|)^{-s}, m, n \in \mathbb{N}$,

$\left(\mathcal{P}_{2}\right): \exists s>0 \exists C_{s}>0:\left|\left\langle g_{m}, g_{n}\right\rangle\right| \leq C_{s} \mathrm{e}^{-s|m-n|}, m, n \in \mathbb{N}$,

then the property $\mathcal{P}_{\left(g_{n}\right)}$ is satisfied.

Now we apply Theorem 4.1 to obtain series expansions in the spaces $\mathcal{S}$ and $\Sigma^{\alpha}$ (for $\alpha>1 / 2$ ), and their duals, via frames localized by the Hermite orthonormal basis and coefficients in the corresponding sequence spaces. Furthermore, we extend the known characterizations of $\mathcal{S}, \Sigma^{\alpha}, \alpha>1 / 2$, and their dual spaces, based on the Hermite basis (see the end of Sec. 2), to characterizations based on a larger class of frame-functions.

Theorem 4.2 Assume that $\left(e_{n}\right)_{n=1}^{\infty}$ is a sequence with elements from $\mathcal{S}(\mathbb{R})$ which is a frame for $L^{2}(\mathbb{R})$ and which is polynomially localized with respect to the Hermite basis $\left(h_{n}\right)_{n=1}^{\infty}$ with decay $s$ for every $s \in \mathbb{N}$. Take $\left(g_{n}\right)_{n=1}^{\infty}:=\left(h_{n}\right)_{n=1}^{\infty}$. Then $\mathcal{P}_{\left(g_{n}\right)}$ and the conclusions in Theorem 4.1 hold with $X_{F}$ replaced by $\mathcal{S}$ and $\Theta_{F}$ replaced by s.

Theorem 4.3 Let $\alpha>1 / 2$. Assume that a sequence $\left(e_{n}\right)_{n=1}^{\infty}$ with elements from $\Sigma^{\alpha}$ is a frame for $L^{2}(\mathbb{R})$ which is exponentially localized with respect to the Hermite basis $\left(h_{n}\right)_{n=1}^{\infty}$. Take $\left(g_{n}\right)_{n=1}^{\infty}:=\left(h_{n}\right)_{n=1}^{\infty}$. Then $\mathcal{P}_{\left(g_{n}\right)}$ and the conclusions in Theorem 4.1] hold with $X_{F}$ replaced by $\Sigma^{\alpha}$ and $\Theta_{F}$ replaced by $\mathfrak{s}^{1 /(2 \alpha)}$.

To illustrate Theorems 4.2 and 4.3 , below we give an example based on appropriate linear combinations of Hermite functions. Further examples are to be given in an extended paper [23].

Example 4.4 Let $r \in \mathbb{N}$ and for $i=1,2, \ldots, r$, take $\varepsilon_{i} \geq 0$ and a sequence $\left(a_{n}^{i}\right)_{n=1}^{\infty}$ of complex numbers satisfying $\left|a_{n}^{i}\right| \leq \varepsilon_{i}$ for $n \geq 2, \sum_{i=1}^{r}\left|a_{1}^{i}\right| \leq 1$, and $\sum_{i=1}^{r} \varepsilon_{i}<1$. For $n \in \mathbb{N}$, consider $e_{n}:=h_{n}+\sum_{i=1}^{r} a_{n}^{i} h_{n+i}$, which clearly belongs to $\mathcal{S}(\mathbb{R})$ and $\Sigma^{\alpha}$, $\alpha>1 / 2$. Then the sequence $\left(e_{n}\right)_{n=1}^{\infty}$ is a Riesz basis for $L^{2}(\mathbb{R})$ and it is s-localized with respect to the Hermite orthonormal basis $\left(h_{n}\right)_{n=1}^{\infty}$ for every $s>0$, as well as exponentially localized with respect to $\left(h_{n}\right)_{n=1}^{\infty}$. 
Remark 4.5 Having in mind the known expansions of tempered distributions $\left(\mathcal{S}\left(\mathbb{R}_{+}\right)\right)^{\prime}$ [25, 9] and Beurling ultradistributions $\left(G_{\alpha}^{\alpha}\left(\mathbb{R}_{+}\right)\right)^{\prime}$ [10, 18], and their test spaces, by the use of the Laguerre orthonormal basis $l_{n}, n \in \mathbb{N}$, and validity of the corresponding properties $\mathcal{P}_{\left(l_{n}\right)}$, we can transfer the above results to the mentioned classes of distributions and ultradistributions over $\mathbb{R}_{+}$.

Acknowledgements The authors acknowledge support from the Multilateral S\&T Danube-Cooperation Project TIFMOFUS ("Time-Frequency Methods for Operators and Function Spaces"; MULT_DR 01/2017), the Austrian Science Fund (FWF) START-project FLAME ('Frames and Linear Operators for Acoustical Modeling and Parameter Estimation'; Y 551-N13), and the Project 174024 of the Serbian Ministry of Sciences. The second author is grateful for the hospitality of the University of Novi Sad, where most of the research on the presented topic was done.

\section{References}

[1] A. Aldroubi, Q. Sun, W. Tang. $p$-frames and shift invariant subspaces of $L^{p}$. J. Fourier Anal. Appl. 7(1), 1-21 (2001). [3]

[2] R. Balan, P. G. Casazza, C. Heil, Z. Landau. Density, overcompleteness, and localization of frames. I: Theory. J. Fourier Anal. Appl. 12(2), 105-143 (2006).

[3] R. Balan, P. G. Casazza, C. Heil, Z. Landau. Density, overcompleteness, and localization of frames. II: Gabor systems. J. Fourier Anal. Appl. 12(3), 307344 (2006).

[4] N. K. Bari, Biorthogonal systems and bases in Hilbert space. Mathematics. Vol. IV, Uch. Zap. Mosk. Gos. Univ., 148, Moscow Univ. Press, Moscow, 1951, 69-107.

[5] P. Casazza, O. Christensen, D. T. Stoeva. Frame expansions in separable Banach spaces, J. Math. Anal. Appl. 307, 710-723 (2005).

[6] Y. Chen, M. Signahl, J. Toft: Factorizations and singularvalue estimates of operators with Gelfand-Shilov and Pilipovic' kernels. J. Fourier Anal. Appl. to appear

[7] O. Christensen, An Introduction to Frames and Riesz Bases. Second Expanded Edition, Series: Applied and Numerical Harmonic Analysis, Birkhäuser, Boston, 2016.

[8] R. J. Duffin, A. C. Schaeffer, A class of nonharmonic Fourier series. Trans. Am. Math. Soc. 72, 341-366 (1952).

[9] A. J. Duran: Laguerre expansions of tempered distributions and generalized functions. J. Math. Anal. Appl. 150(1), 166-180 (1990).

[10] A. J. Duran: Laguerre expansions of Gel'fand-Shilov spaces, J. Approx. Theory 74, 280-300 (1993). 
[11] C. Fernandez, A. Galbis Verdu, J. Toft: The Bargmann transform and powers of harmonic oscillator on Gelfand-Shilov subspaces. Revista de la Real Academia de Ciencias Exactas, Fsicas y Naturales. Serie A. Matemticas. 111, 1-13 (2017).

[12] F. Futamura: Banach framed, decay in the context of localization, Sampl. Theory Signal Image Process. 6(2), 151-166 (2007).

[13] M. Fornasier, K. Gröchenig: Intrinsic localization of frames. Constructive Approximation 22(3), 395-415 (2005).

[14] H. G. Feichtinger, K. Grochenig. Banach Spaces Related to Integrable Group Representations and Their Atomic Decompositions. I. J. Funct. Anal. 86(2), 307-340 (1989).

[15] H. G. Feichtinger, K. Grochenig. Banach Spaces Related to Integrable Group Representations and Their Atomic Decompositions II. Monatsh. Math. 108(23), 129-148 (1989).

[16] K. Gröchenig, Describing functions: atomic decompositions versus frames. Monatsh. Math. 112(1), 1-42 (1991).

[17] K. Gröchenig: Localization of frames, Banach frames, and the invertibility of the frame operator. J. Fourier Anal. Appl. 10(2), 105-132 (2004).

[18] S. Jakšić, S. Pilipović, B. Prangoski: G-type spaces of ultradistributions over $\mathbb{R}_{+}^{d}$ and the Weyl pseudo-differential operators with radial symbols. Revista de la Real Academia de Ciencias Exactas, Fsicas y Naturales. Serie A. Matemticas 111(3), 613-640 (2017).

[19] S. Pilipović: Tempered ultradistributions. Boll. Unione Mat. Ital., 7(2-B), 235-251 (1988).

[20] S. Pilipović, D. T. Stoeva, N. Teofanov: Frames for Fréchet spaces. Bull. $C l$. Sci. Math. Nat. Sci. Math., 32, 69-84, 2007.

[21] S. Pilipović, D. T. Stoeva: Series expansions in Fréchet spaces and their duals, construction of Fréchet frames. J. Approx. Theory 163, 1729-1747 (2011).

[22] S. Pilipović, D. T. Stoeva: Fréchet frames, general definition and expansions. Anal. Appl. 12(2), 195-208 (2014).

[23] S. Pilipović, D. T. Stoeva: Weighted localization of frames and the expansions of distributions and ultradistributions. In preparation.

[24] B. Simon, Distributions and their Hermite expansions, J. Math. Phys. 12(1), 140-148 (1971).

[25] M. Guillemot-Teissier: Développements des distributions en séries de fonctions orthogonales. Séries de Legendre et de Laguerre (French). (Development of distributions in series of orthogonal functions. Series of Legendre an Laguerre). Ann. Sc. Norm. Super. Pisa, Sci. Fis. Mat., III. Ser. 25, 519-573 (1971). 